\title{
Saúde mental da equipe multiprofissional de Centro de Atenção Psicossocial durante enfrentamento de crises inerentes ao trabalho
}

Mental health of the multidisciplinary team of the Psychosocial Care Center during coping with work-related crises

Salud mental del equipo multidisciplinar del Centro de Atención Psicosocial ante crisis inherentes al trabajo

Recebido: 18/08/2021 | Revisado: 29/08/2021 | Aceito: 30/08/2021 | Publicado: 01/09/2021

\author{
Solange Cristina Denzin Rosa \\ ORCID: https://orcid.org/0000-0002-8083-881X \\ Centro de Atenção Psicossocial AD, Brasil \\ E-mail: solangecdr@gmail.com \\ Maria José dos Reis \\ ORCID: https://orcid.org/0000-0003-2717-2163 \\ Universidade Estadual de Campinas, Brasil \\ E-mail: mjreis03@hotmail.com \\ Maria Neyrian de Fátima Fernandes \\ ORCID: https://orcid.org/0000-0001-7626-9733 \\ Universidade Federal do Maranhão, Brasil \\ E-mail: neyrian.maria@ufma.brr \\ Mônica Chaves \\ ORCID: https://orcid.org/0000-0002-5026-6558 \\ Pontifícia Universidade Católica de Minas Gerais, Brasil \\ E-mail: monikachaves@uol.com.br \\ Rafael Braga Esteves \\ ORCID: https://orcid.org/0000-0003-4604-6840 \\ Universidade de São Paulo, Brasil \\ E-mail: rafael.braga.esteves@alumni.usp.br
}

\section{Resumo}

Analisar as características de crises que podem ocorrer com a equipe multiprofissional que atua no Centro de Atenção Psicossocial (CAPS) e, o quanto isso pode afetar a saúde mental e as atividades laborais diárias desses profissionais. Trata-se de um ensaio teórico e reflexivo com características descritivas, proposto após a observação de dinâmicas nas práticas dos serviços de saúde mental. A reflexão foi baseada nos resultados de buscas na legislação brasileira que rege a assistência à saúde mental, de artigos de periódicos especializados em ciências da saúde em geral, saúde mental, psiquiatria e saúde públicas, além de livros nas áreas descritas e de autores considerados especialista com ampla produção teórico-cientifica. Os profissionais tornam-se vulneráveis a eventos estressores não resolvidos no local de trabalho, assim o adoecimento mental é uma possibilidade quando os gestores e a sociedade não percebem as necessidades dos trabalhadores, ignorando suas queixas e apontamentos. Nas situações em que algum membro da equipe multiprofissional demonstra atitudes e comportamentos negativos, possivelmente estará afetando os demais colegas, criando um ambiente nocivo e tóxico para a equipe e dificultando o processo de trabalho para a recuperação do paciente e família. Ainda são necessários mais estudos e pesquisas nesta área, visto que poucos artigos enfatizaram a saúde mental da equipe do CAPS, pouco se escreve sobre o sofrimento dos profissionais que atuam em unidades de saúde mental. Há pouca valorização sobre este tema, implícito está que os trabalhadores da saúde não adoecem, nem tem necessidades, apenas assistem ao paciente em sofrimento.

Palavras-chave: Centros comunitários de saúde mental; Pessoal de saúde; Riscos ocupacionais; Sistema único de saúde.

\footnotetext{
Abstract

To analyze the characteristics of crises that can occur with the multidisciplinary team that works at the Psychosocial Care Center (CAPS) and how much this can affect the mental health and daily work activities of these professionals. This is a theoretical and reflective essay with descriptive characteristics, proposed after the observation of dynamics in the practices of mental health services. The reflection was based on the results of searches in Brazilian legislation governing mental health care, articles from journals specializing in health sciences in general, mental health, psychiatry and public health, as well as books in the areas described and authors, considered specialists. with
} 
extensive theoretical and scientific production. Professionals become vulnerable to unresolved stressful events in the workplace, so mental illness is a possibility when managers and society do not realize the workers' needs, ignoring their complaints and notes. In situations where a member of the multidisciplinary team demonstrates negative attitudes and behaviors, it is likely affecting other colleagues, creating a harmful and toxic environment for the team and hindering the work process for the recovery of the patient and family. More studies and research in this area are still needed, as few articles have emphasized the mental health of the CAPS team, little is written about the suffering of professionals working in mental health units. There is little appreciation of this topic, it is implicit that health workers do not get sick, nor do they have needs, just assist the patient in distress.

Keywords: Community mental health centers; Health personnel; Occupational risks; Unified health system.

\section{Resumen}

Analizar las características de las crisis que pueden ocurrir con el equipo multidisciplinar que trabaja en el Centro de Atención Psicosocial (CAPS) y cuánto puede afectar esto a la salud mental y la actividad laboral diaria de estos profesionales. Se trata de un ensayo teórico y reflexivo con características descriptivas, propuesto tras la observación de dinámicas en las prácticas de los servicios de salud mental. La reflexión se basó en los resultados de búsquedas en la legislación brasileña que regula la atención en salud mental, artículos de revistas especializadas en ciencias de la salud en general, salud mental, psiquiatría y salud pública, así como libros en las áreas descritas y autores considerados especialistas. producción teórica y científica. Los profesionales se vuelven vulnerables a eventos estresantes no resueltos en el lugar de trabajo, por lo que la enfermedad mental es una posibilidad cuando los gerentes y la sociedad no se dan cuenta de las necesidades de los trabajadores, ignorando sus quejas y notas. En situaciones en las que un miembro del equipo multidisciplinario demuestra actitudes y comportamientos negativos, es probable que esté afectando a otros compañeros, creando un ambiente nocivo y tóxico para el equipo y dificultando el proceso de trabajo para la recuperación del paciente y su familia. Aún se necesitan más estudios e investigaciones en esta área, ya que pocos artículos han enfatizado la salud mental del equipo CAPS, poco se escribe sobre el sufrimiento de los profesionales que trabajan en las unidades de salud mental. Hay poca apreciación de este tema, está implícito que los trabajadores de la salud no se enferman, ni tienen necesidades, solo atienden al paciente en apuros.

Palabras clave: Centros comunitarios de salud mental; Personal de salud; Riesgos laborales; Sistema único de salud.

\section{Introdução}

O presente estudo é um ensaio teórico reflexivo acerca da saúde mental da equipe multiprofissional dos Centros de Atenção Psicossocial (CAPS), considerando importante a contextualização histórica para compreender o desenvolvimento deste modelo de atendimento territorial em saúde mental aos longos dos anos até os dias atuais e apresentado um dos marcos da psiquiatria, a Reforma Psiquiátrica.

A Reforma Psiquiátrica pode ser compreendida como um processo paulatino de mudanças da forma asilar do cuidado em saúde mental para pacientes com transtornos mentais. Esse processo ocorreu no Brasil e foi fortemente influenciado pela Reforma Psiquiátrica italiana, e os pensamentos de Franco Basaglia (Basaglia, 2010; Bueno, 2020; Demarco, Jardim, \& Kantorski, 2016). Diante deste contexto, descrevendo os impactos desta Reforma na atenção à saúde mental e nas políticas de saúde brasileira, destaca-se como marco histórico e legal a Lei n 10.216/2001, "por uma sociedade sem manicômios" com uma lógica inclusiva para os usuários com transtornos mentais (Ministério da Saúde, 2001). Neste sentido, a forma de pensar na saúde mental é legitimada e transformada ao longo dos anos, ocorrendo de uma transição do modelo hospitalocêntrico para um modelo de atenção à saúde descentralizado e territorial. Essas modificações refletiram na redução dos números de leitos hospitalares e em estratégias de reinserção desses pacientes na sociedade (Ministério da Saúde, 1990; 2001).

Os CAPS ofertam serviços territoriais, especializados em saúde mental, objetivando ações de promoção, recuperação e reabilitação da saúde, sendo consonantes com o Sistema Único de Saúde (SUS) (Amarante \& Nunes, 2018; Dias, Ferigato, \& Fernandes, 2020; Jafelice \& Marcolan, 2018). O CAPS foi criado para garantir a atenção a saúde mental e a redução dos números de leitos de hospitais psiquiátricos. Este modelo territorial de atenção à saúde exige da equipe que assiste ao paciente um olhar diferenciado quanto à organização do trabalho e a divisão de tarefas (Garcia, Zanoti-Jeronymo, Zambenedetti, Cervo, \& Cavalcante, 2020; Pinho, Souza, \& Esperidião, 2018). Esta equipe deve ser uma equipe multiprofissional, ou seja, deve ser formada por profissionais de diferentes áreas para atender aos requisitos exigidos de diretrizes postas pelo Ministério da Saúde 
(Ministério da Saúde, 2002; 2004). Todos os membros da equipe devem ter como objetivo prestar uma assistência aos indivíduos com psicopatologias moderadas e graves, buscando a autonomia desse usuário do SUS (Jafelice \& Marcolan, 2018).

O CAPS é um serviço de saúde aberto e comunitário do SUS, isso o caracteriza como um dispositivo de referência e tratamento para pessoas que sofrem com transtornos mentais, incluindo o uso e abuso de álcool e drogas (Ministério da Saúde, 2011). Além dos quadros cuja severidade ou persistência podem justificar a permanência em um dispositivo de cuidado intensivo (Araújo, 2018). Durante o desenvolvimento das atividades, os profissionais da equipe dos CAPS podem vivenciar situações críticas em decorrência das complicações causadas pelo transtorno mental. Essas crises presenciadas e atendidas pela equipe, associadas a outros fatores estressores, podem influenciar na capacidade laboral e no adoecimento físico e mental dos profissionais (Costa, Jorge, Coutinho, Costa, \& Holanda, 2016; Lima \& Dimenstein, 2016; Rossi, Marcolino, Speranza, \& Cid, 2019).

Os profissionais da equipe multiprofissional não lidam apenas com as crises dos usuários dos CAPS, lidam também com as crises entre os próprios membros da equipe nos diversos ambientes de trabalho (Zeferino, Cartana, Fialho, Huber, \& Bertoncello, 2016). Assim, as características do trabalho da equipe multiprofissional dos CAPS merecem atenção dos gestores da saúde pública, em especial daqueles responsáveis por viabilizar os processos e práticas do trabalho de maneira humanizada (Bellenzani, Paro, \& DeOliveira, 2016).

Nesse sentido, estudos demonstram a ocorrência de crises enfrentadas pela equipe multiprofissional dos CAPS, além de apontar estratégias e intervenções que incidem na qualidade do ambiente laboral (Bellenzani, Paro, \& De Oliveira, 2016; Cardoso, Oliveira, \& Piani, 2015). Quando a equipe, por diversas razões, não consegue administrar situações críticas geradas pelos usuários em tratamento ou pelos membros da equipe, observa-se uma tendência negativa que pode afetar o processo do trabalho em grupo. O que muitas vezes se estende à qualidade da assistência prestada aos usuários do serviço em saúde mental do CAPS.

Diante do exposto, é proposto um ensaio teórico e reflexivo, que objetiva analisar as características de crises que podem ocorrer com a equipe multiprofissional que atua no CAPS e, o quanto isso pode afetar a saúde mental e as atividades laborais diárias desses profissionais.

\section{Metodologia}

Trata-se de um ensaio teórico e reflexivo com características descritivas, proposto após a observação de dinâmicas nas práticas dos serviços de saúde mental, abordadas neste texto pelo termo 'crises', usado, frequentemente, dentro das equipes dos CAPS e que se podem causar o adoecimento mental (Marconi \& Lakatos, 2017; Fontelles, Simões, Farias, Garcia, \& Fontelles, 2009). A reflexão foi baseada nos resultados de buscas na legislação brasileira que rege a assistência à saúde mental, de artigos de periódicos especializados em ciências da saúde em geral, saúde mental, psiquiatria e saúde públicas, além de livros nas áreas descritas e de autores considerados especialista com ampla produção teórico-cientifica. Dessa forma, a discussão deste ensaio ocorrerá em três categorias temáticas: 1 . O contexto de trabalho da equipe multiprofissional do Centro de Atenção Psicossocial; 2. Equipe multiprofissional na saúde mental e 3. Saúde mental da equipe e as crises inerentes ao trabalho.

\section{Resultados e Discussão}

\subsection{O contexto de trabalho da equipe multiprofissional do Centro de Atenção Psicossocial}

Nos últimos anos, verificou-se avanços no contexto das políticas públicas de saúde brasileira, fruto de um intenso processo de mobilização social do movimento da reforma sanitária. No escopo das transformações no campo da saúde, entre outros exemplos, destaca-se o movimento da Reforma Psiquiátrica (DePinho et al., 2011). No que se refere aos estigmas da loucura e os desafios políticos neste contexto: 
"Mais do que buscar a aceitação de uma nova política assistencial, o desafio nesse campo é produzir uma nova sensibilidade cultural para com o tema da loucura e do sofrimento psíquico. Trata-se de promover uma desconstrução social dos estigmas e estereótipos vinculados à loucura e à figura do doente mental, substituindo-os por um olhar solidário e compreensivo sobre a diversidade e os descaminhos que a experiência subjetiva pode apresentar, olhar fundado numa atitude de respeito, tolerância e responsabilidade com aqueles que se encontram com a sua normatividade psíquica restringida" (Bezerra., 2007, p. 247).

A desconstrução histórica e social da loucura deu-se com a Reforma Psiquiátrica brasileira, iniciada na década de 1970 com a transição do modelo asilar para o territorial, consolidando-se com a regulamentação dos CAPS em 2002, Portaria n. 336/GM/2002 (Ministério da Saúde, 2002). Os diversos níveis de CAPS obedecem a uma variada conformação profissional e critério populacional conforme as modalidades, sendo: CAPS I, CAPS II, CAPS III, CAPSi e CAPSad, de acordo com a Portaria GM nº. 336/2002 (Ministério da Saúde, 2002; 2004).

Os CAPS são instituições destinadas a acolher os pacientes com transtornos mentais, estimular a sua integração social e familiar, além de subsidiá-los através de estratégias diversas o desenvolvimento de autonomia, provendo atendimento médico e psicológico (Ministério da Saúde, 2004). Dessa forma, foi reformulada a assistência em Saúde Mental, que, para oferecer um tratamento territorial e inclusivo, investiu-se nos "serviços de atenção à crise", também por meio da implementação de CAPS (Dias et al., 2020).

Estes serviços se contrapõem ao modelo hospitalocêntrico e têm como população alvo os pacientes com transtorno mental e comportamental, principalmente, psicoses e neuroses graves (Filizola, Milioni, \& Pavarini, 2008). O cuidado é desenvolvido por intermédio de Projeto Terapêutico Singular (PTS), envolvendo a equipe, o usuário e a sua família. A ordenação do cuidado estará sob a responsabilidade do CAPS e/ou da Atenção Básica (Ministério da Saúde, 2011).

Dessa forma, os CAPS vêm se consolidando como dispositivos eficazes na diminuição de internações em hospitais psiquiátricos, implementando a mudança do modelo assistencial. Para isso, os CAPS devem oferecer diversas atividades terapêuticas, como psicoterapia individual ou em grupo, oficinas diversas, atividades na comunidade, orientação e acompanhamento do uso de medicações, atendimento domiciliar. Estas atividades são organizadas através de PTS, que tem por objetivo o tratamento e a reabilitação para promover a autonomia pessoal e social dos usuários (Araújo, 2018; Hepp, 2013).

Diante do exposto, pode-se perceber que o CAPS é um dispositivo essencial do movimento da reforma psiquiátrica brasileira para romper estigmas sobre a loucura, prover atendimento humanizado, causar mudanças da estrutura das equipes de profissionais da saúde mental e organização do trabalho com uma lógica inclusiva do usuário. Nesse sentido, gerenciar as situações de crises internas enfrentadas pela equipe multiprofissional é fundamental para a qualidade dos serviços de saúde mental.

\subsection{Equipe multiprofissional na saúde mental}

A equipe especializada em saúde mental nos CAPS realiza o acolhimento dos pacientes, podendo ser um substitutivo a internação psiquiátrica. Para obtenção de sucessos nas intervenções realizadas é necessária uma equipe multiprofissional organizada interdisciplinarmente, competente e coesa. Todos os elementos da equipe visam manter as relações de trabalho o mais saudável possível respeitando as diferenças socioculturais de cada profissional de modo a acolher, planejar e implementar o tratamento psiquiátrico ao paciente em um ambiente acolhedor e seguro.

Os profissionais de nível superior que atuam nos CAPS são: enfermeiros, médicos, psicólogos, assistentes sociais, terapeutas ocupacionais, pedagogos, professores de educação física ou outros necessários para as atividades oferecidas nos CAPS. Os profissionais de nível médio podem ser: técnicos e/ou auxiliares de enfermagem, técnicos administrativos, educadores e artesãos. Os CAPS contam ainda com equipes de limpeza e de cozinha (Ministério da Saúde, 2002; 2004). 
O serviço com o modelo de atenção psicossocial requer nova abordagem dos profissionais para o cuidado em saúde mental. Ainda, nesse ambiente de trabalho ocorre intensa interação entre profissional e o usuário do serviço, sendo essa uma característica mais humanizada, mas também mais desgastante para o profissional. Nessa interação, a equipe multiprofissional no trabalho deve buscar a articulação das ações, para uma concepção menos conflituosa (Gonçalves, Vilela, Terra, \& Nogueira, 2016; Laurentino, Pereira, Barbosa, \& Maia, 2017). Portanto, ao definirmos como meta o trabalho em equipe nos CAPS, não deve haver a pressuposição idealizada de igualdade de saberes técnicos e valores sociais dos diferentes trabalhos profissionais, mas antes o enfrentamento dessas diferenças e desigualdades no processo cotidiano de trabalhar/cuidar (Martins, 2017).

Ao considerar essa nova organização do trabalho os profissionais estão sujeitos a experienciar diversas situações de crise, visto que as características dos serviços demandam uma maior proximidade entre os usuários dos serviços e os profissionais da equipe.

\subsection{Saúde mental da equipe e as crises inerentes ao trabalho}

A saúde mental da equipe multiprofissional do CAPS é pouco trabalhada, apesar da unidade pressuposta, cada profissional desempenha papéis diferentes, porém significativos no cuidado ao indivíduo em sofrimento mental de modo a atendê-los em suas necessidades, além de acolher a família do paciente. Considera-se que cada componente da equipe atue com o objetivo de atender às demandas do paciente. Entretanto, pesquisas que analisaram o trabalho em equipes de saúde mental nos novos dispositivos de atenção em saúde mental vêm apontando tensões existentes na delimitação do saber e de campos específicos do conhecimento (Jafelice \& Marcolan, 2018; Martins, 2017).

Estas tensões são geradas pela diversidade de situações que aparecem no contexto do ambiente de trabalho da equipe, nas quais as intervenções são realizadas em situações de crises apresentadas por alguns usuários do serviço. Estas situações de crise podem influenciar o equilíbrio do trabalho multiprofissional, acrescentando outros componentes estressores, diversos e inesperados, a conflitos preexistentes das equipes. A crise é considerada como uma condição que envolve muito mais a dimensão social do que as dimensões biológica e psicológica, podendo denunciar a pouca solidariedade entre as pessoas ou uma situação precária no que se refere à existência de recursos para tratamento no território ou no domicilio (Amarante, 2008). Assim, podemos dizer que originalmente a palavra crise está carregada de elementos que trazem um amplo sentido, separação, mudança, desequilíbrio transitório, com possível adaptação (Dias et al., 2020).

Os profissionais, muitas vezes, reconhecem seu despreparo para atender as situações de crise, gerando neles mesmos insegurança, medo e dificuldades para desenvolver o trabalho em uma instituição designada para atender um público em situação de crise. Todavia, a crise não atinge apenas o usuário que sofre intensamente com a falta do acolhimento, mas também à saúde mental dos profissionais (Cruz, Guerrero, \& Vieira, 2019; Krachenski \& Holanda, 2019).

Apesar da equipe de profissionais terem as competências e as habilidades necessárias para o exercício das funções no atendimento ao indivíduo com adoecimento mental, esses profissionais também podem demostrar sinais de estresse ao longo dos anos de trabalho na área da saúde mental. Muitas vezes a própria equipe age de forma a alertar sobre a iminência de "crise" entre seus membros, observa-se o cansaço físico, discursos de descontentamento com o serviço, prejuízo na memória ou mesmo desentendimentos na atuação de cada membro do grupo de profissionais (Zeferino et al., 2016; Moreira \& Lucca, 2020).

Quando a organização do processo de trabalho é precária ao ponto de desequilibrar a saúde, o trabalhador direciona suas forças para encontrar estratégias de defesa e lidar com o sofrimento (Araújo, 2018). De modo geral, a população procura pelos serviços prestados no CAPS com a expectativa de receber acolhimento. E também um atendimento humanizado, congruentes com as suas necessidades. No entanto, é rara a percepção de que a equipe multiprofissional também necessita de 
atendimento e cuidado conforme as necessidades para manter a saúde mental de seus membros (Coelho \& Scatolin, 2020; Pinho et al., 2018).

O adoecimento da equipe sem um suporte técnico é real e poderá influenciar a dinâmica dos atendimentos e o relacionamento entre seus membros. $\mathrm{O}$ adoecimento psíquico relacionado ao trabalho é, atualmente, um enorme desafio com o qual se defrontam os profissionais dedicados ao cuidado da saúde (Pereira et al., 2020). O aumento do número de registro de doenças relacionadas ao trabalho a cada ano instiga os pesquisadores a investigar a relação entre o surgimento de doenças (físicas, mentais ou psicossomáticas) e a organização do trabalho. Lidar com as crises exige mais preparo, coisa que os profissionais alegam não possuir, o que demonstra a necessidade de investimento na capacitação dos recursos humanos já existentes e na seleção de profissionais para trabalhar nos CAPS (Costa et al., 2016; Roquette, 2019).

$\mathrm{Na}$ literatura especializada, observa-se que há poucas ações voltadas à saúde do trabalhador de saúde pública como capacitações, reuniões de equipe, redução de jornada de trabalho, supervisão e exercícios de saúde laboral (Ramminger, Silva, \& Freire, 2015). Assim, o atendimento às crises dos usuários sem apoio dos gestores, sem suporte técnico e sem equipamentos para estruturar os projetos desenvolvidos é sem dúvida falar de um serviço fragilizado tendendo a problemas no relacionamento entre os componentes da equipe incluindo aqueles que da equipe dependem para atingir o equilíbrio psíquico (Matias, Carvalho, Silva, \& Lopes, 2021).

A Política Nacional de Humanização (PNH), defende a humanização da atenção à saúde dos usuários do SUS, destacando que isso não acontece dissociado das questões concernentes ao bem-estar das equipes e das condições de trabalho. Ou seja, não é possível pensar na melhoria do modelo de atendimento ao usuário, mais humanizado, desagregado da necessidade de constituição de relações também humanizadas entre as equipes e nos níveis de gestão (Bellenzani et al., 2016). As ações de promoção à saúde poderão auxiliar os trabalhadores a desenvolver práticas de autocuidado e a enfrentar as demandas e os estressores inerentes ao processo de trabalho. Para os trabalhadores dos CAPS, destaca-se que oportunizar melhorias na qualidade de vida no trabalho pode representar uma redução do índice de absenteísmo por doenças ocupacionais, bem como melhor qualidade de vida (Bellenzani et al., 2016; Bitencourt Toscani Greco et al., 2019; Ministério da Saúde, 2015).

\section{Considerações Finais}

Os profissionais tornam-se vulneráveis a eventos estressores não resolvidos no local de trabalho, assim o adoecimento mental é uma possibilidade quando os gestores e a sociedade não percebem as necessidades dos trabalhadores, ignorando suas queixas e apontamentos. Nas situações em que algum membro da equipe multiprofissional demonstra atitudes e comportamentos negativos, possivelmente estará afetando os demais colegas, criando um ambiente nocivo e tóxico para a equipe e dificultando o processo de trabalho para a recuperação do paciente e família.

Ainda são necessários mais estudos e pesquisas nesta área, visto que poucos artigos enfatizaram a saúde mental da equipe do CAPS, pouco se escreve sobre o sofrimento dos profissionais que atuam em unidades de saúde mental. Há pouca valorização sobre este tema, implícito está que os trabalhadores da saúde não adoecem, nem tem necessidades, apenas assistem ao paciente em sofrimento.

A reforma psiquiátrica aconteceu para dar esperanças aos indivíduos que vivenciam o adoecimento mental, dar-lhes a possibilidade de terem uma vida sem preconceitos, receberem o tratamento o mais próximo possível das suas comunidades. Por isso, essa assistência não deve proporcionar crise e cansaço para os profissionais que a provê para esses usuários. É importante entender que estes profissionais necessitam de estratégias de promoção da saúde, somente assim evitarão doenças ocupacionais mantendo sua plena capacidade para o trabalho. 


\section{Referências}

Amarante, P., \& Nunes, M. de O. (2018). Psychiatric reform in the SUS and the struggle for a society without asylums. Ciencia e Saude Coletiva, 23(6), 20672074. https://doi.org/10.1590/1413-81232018236.07082018.

Araújo, C. S. B. (2018). Processo de trabalho em saúde mental: um estudo com trabalhadores de CAPS. Dissertação (Mestrado em Geografia). Universidade Federal de Uberlândia, Uberlândia. https://doi.org/10.14393/ufu.di.2018.956.

Basaglia, F. (2010). Escritos Selecionados em Saúde Mental e Reforma Psiquiátrica. Rio de Janeiro: Garamond.

Bellenzani, R., Paro, D. M., \& DeOliveira, M. C. (2016). Trabalho em Saúde Mental e Estresse na Equipe: Questões para a Política Nacional de Humanização/SUS. Revista Psicologia e Saúde, 8(1), 32-43. https://doi.org/10.20435/2177093x2016105.

Bezerra Jr., B. (2007). Desafios da reforma psiquiátrica no Brasil. Physis: Revista de Saúde Coletiva, 17(2), 243-250. https://doi.org/10.1590/S010373312007000200002 .

Bitencourt Toscani Greco, P., Morozini Dias, A., Soares Bernardi, C. M., Moraes Legramante, D., Mancio Ferreira da Luz, E., Mazzuco de Souza, M., \& Dal Ongaro, J. (2019). Promoção à saúde com trabalhadores de saúde mental. Revista Brasileira em Promoção da Saúde, 32(0), 1-9. https://doi.org/10.5020/18061230.2019.9669.

Bueno, R. C. (2020). O pensamento de Franco Basaglia: dos caminhos da saúde mental italiana a uma vivência prática em Trieste (1st ed.). Belo Horizonte: Editora Dialética.

Cardoso, M. R. de O., Oliveira, P. de T. R. de, \& Piani, P. P. F. (2015). Relato de experiência de um atendimento em um CAPS: considerações sobre o cuidado em saúde mental. Rev. NUFEN.

Coelho, T. M., \& Scatolin, H. G. (2020). Os riscos psicossociais relacionados com o trabalho: Impactos na subjetividade do trabalhador. Revista Científica Multidisciplinar Núcleo do Conhecimento, 122-138. https://doi.org/10.32749/nucleodoconhecimento.com.br/psicologia/riscos-psicossociais.

Costa, J. P., Jorge, M. S. B., Coutinho, M. P. de L., Costa, E. C., \& Holanda, Í. T. A. (2016). A reforma psiquiátrica e seus desdobramentos: representações sociais dos profissionais e usuários da atenção psicossocial. Psicologia e Saber Social, 5(1), 35-45. https://doi.org/10.12957/psi.saber.soc.2016.15855.

Cruz, K. D. F. da, Guerrero, A. V. P., \& Vieira, J. S. N. (2019). Atenção à crise em saúde mental: um desafio para a reforma psiquiátrica brasileira. Revista do NUFEN, 11(2), 117-132. https://doi.org/10.26823/REVISTADONUFEN.VOL11.N02ENSAIO51.

Demarco, D. de A., Jardim, V. M. da R., \& Kantorski, L. P. (2016). Cuidado em saúde às pessoas com transtorno mental na Rede de Atenção Psicossocial Health care to people with mental disorders in the network for psychosocial care. Revista de Pesquisa Cuidado é Fundamental Online, 8(3), $4821-4825$. https://doi.org/10.9789/2175-5361.2016.v8i3.4821-4825.

DePinho, L. B., Kantorski, L. P., Wetzel, C., Schwartz, E., Lange, C., \& Zillmer, J. G. V. (2011). Avaliação qualitativa do processo de trabalho em um centro de atenção psicossocial no Brasil. Revista Panamericana de Salud Publica, 30(4), 354-360. https://doi.org/10.1590/S1020-49892011001000009.

Dias, M. K., Ferigato, S. H., \& Fernandes, A. D. S. A. (2020). Attention to the crisis in mental health: Centralization and decentralization of practices. Ciencia e Saude Coletiva, 25(2), 595-602. https://doi.org/10.1590/1413-81232020252.09182018.

Fontelles, M. J., Simões, M. G., Farias, S. H., Garcia, R., \& Fontelles, S. (2009). Metodologia da Pesquisa Científica: diretrizes para elaboração de um protocolo de pesquisa. Núcleo de Bioestatística Aplicado à Pesquisa Da Universidade Da Amazonia - UNAMA, 8. Retrieved from http://files.bvs.br/upload/S/0101-5907/2009/v23n3/a1967.pdf.

Garcia, G. D. V., Zanoti-Jeronymo, D. V., Zambenedetti, G., Cervo, M. da R., \& Cavalcante, M. D. M. A. (2020). Healthcare professionals' perception of mental health in primary care. Revista Brasileira de Enfermagem, 73(1), e20180201. https://doi.org/10.1590/0034-7167-2018-02011.

Gonçalves, A. M., Vilela, S. de C., Terra, F. de S., \& Nogueira, D. A. (2016). Atitudes e o prazer/sofrimento no trabalho em saúde mental. Revista Brasileira de Enfermagem, 69(2), 266-274. https://doi.org/10.1590/0034-7167.2016690209I.

Hepp, C. (2013). Crise Na Saúde Mental: Visão da Equipe. http://www.univates.br/bdu.

Jafelice, G. T., \& Marcolan, J. F. (2018). The multiprofessional work in the Psychosocial Care Centers of São Paulo State. Revista Brasileira de Enfermagem, 71(suppl 5), 2131-2138. https://doi.org/10.1590/0034-7167-2017-0300.

Krachenski, N. B., \& Holanda, A. F. (2019). Manejo de Crise nos Centros de Atenção Psicossocial: Uma Revisão Sistemática de Literatura Crises Management in Psychosocial Care Centers: a Systematic. Pluralidades Em Saúde Mental, 8(1), $23-42$.

Laurentino, S., Pereira, B., Barbosa, J., \& Maia, D. (2017). A política de saúde mental no Piauí sob a égide da RAPS. https://www.ufpi.br/arquivos_download/arquivos/EDUFPI/A_POLÍTICA_DE_SAÚDE_MENTAL_NO_PIAUÍ_SOB_A_ÉGIDE_DA_RAPS.pdf.

Lima, M., \& Dimenstein, M. (2016). O apoio matricial em saúde mental: Uma ferramenta apoiadora da atenção à crise. Interface: Communication, Health, Education, 20(58), 625-635. https://doi.org/10.1590/1807-57622015.0389.

Marconi, M. de A., \& Lakatos, E. M. (2017). Fundamentos de Metodologia Científica (8th ed.). São Paulo: Atlas.

Martins, A. G. (2017). A noção de crise no campo da saúde mental: saberes e práticas em um centro de atenção psicossocial. Mental, 11(20), 226-242. http://pepsic.bvsalud.org/scielo.php?script=sci_arttext\&pid=S1679-44272017000100012\&lng=pt\&tlng=pt.

Matias, C. R. da S., Carvalho, S. G. de, Silva, J. L. D. da, \& Lopes, L. G. F. (2021). Atuação do Enfermeiro no Centro de Atenção Psicossocial. In Gerenciamento de Serviços de Saúde e Enfermagem 3 (pp. 174-183). Atena Editora. https://doi.org/10.22533/at.ed.64221270118. 
Ministério da Saúde. (1990). Lei n ${ }^{\circ}$ 8.080, de 19 de setembro de 1990. Dispõe sobre as condições para a promoção, proteção e recuperação da saúde, organização e o funcionamento dos serviços correspondentes e das outras providências. Diário Oficial da União, Brasília, 1990. http://www.portal.saude.gov.br/portal/arquivos/pdf/LEI8080.pdf.

Ministério da Saúde. (2001). Lei $\mathrm{n}^{\circ}$. 10.216, de 6 de abril de 2001. Dispõe sobre a proteção e os direitos das pessoas portadoras de transtornos mentais e redireciona o modelo assistencial em saúde mental. Diário Oficial da União, Brasília, 2001. http://www.planalto.gov.br/ccivil_03/leis/leis_2001/110216.htm.

Ministério da Saúde. (2002). Portaria GM/MS n 336, de 19 de fevereiro de 2002. Estabelece que os Centros de Atenção Psicossocial poderão constituir-se nas seguintes modalidades de serviços: CAPS I, CAPS II e CAPS III, definidos por ordem crescente de porte/complexidade e abrangência populacional. Diário Oficial da União, Brasília, 2002. http://bvsms.saude.gov.br/bvs/saudelegis/gm/2002/prt0336_19_02_2002.html.

Ministério da Saúde. (2004). Secretaria de Atenção à Saúde. Departamento de Ações Programáticas Estratégicas. Saúde mental no SUS: os centros de atenção psicossocial. http://www.ccs.saude.gov.br/saude_mental/pdf/sm_sus.pdf.

Ministério da Saúde. (2010). Portaria n 4.279, de 30 de dezembro de 2010. Estabelece diretrizes para a organização da Rede de Atenção à Saúde no âmbito do Sistema Único de Saúde (SUS). Diário Oficial da União, Brasília, 2010. https://bvsms.saude.gov.br/bvs/saudelegis/gm/2010/prt4279_30_12_2010.html.

Ministério da Saúde. (2011). Portaria GM/MS n 3.088 , de 23 de dezembro de 2011. Republicada em 21 de maio de 2013 . Institui a Rede de Atenção Psicossocial para pessoas com sofrimento ou transtorno mental, incluindo aquelas com necessidades decorrentes do uso de crack, álcool e outras drogas, no âmbito do Sistema Único de Saúde (SUS). Brasília, 2011. http://bvsms.saude.gov.br/bvs/saudelegis/gm/2011/prt3088_23_12_2011_rep.html.

Ministério da Saúde. (2015). Cadernos Humanizados SUS - Saude mental. In Cadernos Humanizados SUS (V. 5). http://redehumanizasus.net/wpcontent/uploads/2017/09/Cadernos-HumanizaSUS-Volume-5-Saude-Mental.pdf\%0Ahttp://bvsms.saude.gov.br/bvs/publicacoes/saude_mental_volume_5.pdf.

Ministério da Saúde. (2017). Resolução no 32, de 14 de dezembro de 2017. Estabelece as Diretrizes para o Fortalecimento da Rede de Atenção Psicossocial (RAPS). Diário Oficial da União, Brasília, 2017. https://bvsms.saude.gov.br/bvs/saudelegis/cit/2017/res0032_22_12_2017.html.

Moreira, A. S., \& Lucca, S. R. de. (2020). Psychosocial factors and Burnout Syndrome among mental health professionals. Revista Latino-Americana de Enfermagem, 28, 1-11. https://doi.org/10.1590/1518-8345.4175.3336.

Pereira, S. de S., Nascimento, M. M. do, Antonio-Viegas, M. C. R., Morero, J. A. P., Esteves, R. B., Preto, V. A., \& Cardoso, L. (2020). Exaustão emocional em profissionais da saúde e sua associação com variáveis interventoras. Research, Society and Development, 9(7), e877974484. https://doi.org/10.33448/rsdv9i7.4484.

Pinho, E. S., Souza, A. C. S., \& Esperidião, E. (2018). Processos de trabalho dos profissionais dos centros de atenção psicossocial: Revisão integrativa. Ciencia e Saude Coletiva, 23(1), 141-152. https://doi.org/10.1590/1413-81232018231.08332015.

Ramminger, T., Silva, G. da C., \& Freire, F. H. M. de A. (2015). Gênese da Rede de Atenção Psicossocial no Município de Volta Redonda-RJ: um olhar pela narrativa dos trabalhadores. Ayvu: Revista de Psicologia, 1(2), 166. https://doi.org/10.22409/ayvu.v1i2.22186.

Roquette, R. D. (2019). A atenção à crise em saúde mental: refletindo sobre as práticas, a organização do cuidado e os sentidos da crise. (Fundação Oswaldo Cruz). Fundação Oswaldo Cruz. https://www.arca.fiocruz.br/bitstream/icict/39668/2/ve_Rebecca_Dorneles_ENSP_2019.

Rossi, L. M., Marcolino, T. Q., Speranza, M., \& Cid, M. F. B. (2019). Crisis and mental health in adolescence: the story from the perspective of those who live it. Cadernos de Saude Publica, 35(3), e00125018. https://doi.org/10.1590/0102-311x00125018.

Zeferino, M. T., Cartana, M. do H. F., Fialho, M. B., Huber, M. Z., \& Bertoncello, K. C. G. (2016). Health workers' perception on crisis care in the Psychosocial Care Network. Escola Anna Nery, 20(3). https://doi.org/10.5935/1414-8145.20160059. 